
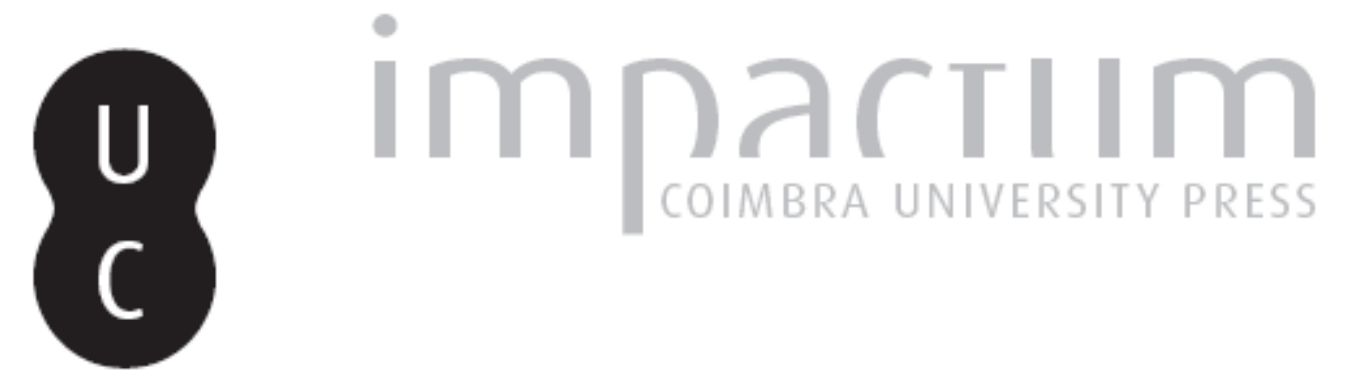

\title{
Le remodelage politique de l'oikos dans la République
}

\author{
Autor(es): Helmer, Étienne
}

Publicado por: Imprensa da Universidade de Coimbra

URL persistente:

URI:http://hdl.handle.net/10316.2/42185

DOI:

DOI:https://doi.org/10.14195/2183-4105_11_6

Accessed : $\quad$ 26-Apr-2023 15:21:51

A navegação consulta e descarregamento dos títulos inseridos nas Bibliotecas Digitais UC Digitalis, UC Pombalina e UC Impactum, pressupõem a aceitação plena e sem reservas dos Termos e Condições de Uso destas Bibliotecas Digitais, disponíveis em https://digitalis.uc.pt/pt-pt/termos.

Conforme exposto nos referidos Termos e Condições de Uso, o descarregamento de títulos de acesso restrito requer uma licença válida de autorização devendo o utilizador aceder ao(s) documento(s) a partir de um endereço de IP da instituição detentora da supramencionada licença.

Ao utilizador é apenas permitido o descarregamento para uso pessoal, pelo que o emprego do(s) título(s) descarregado(s) para outro fim, designadamente comercial, carece de autorização do respetivo autor ou editor da obra.

Na medida em que todas as obras da UC Digitalis se encontram protegidas pelo Código do Direito de Autor e Direitos Conexos e demais legislação aplicável, toda a cópia, parcial ou total, deste documento, nos casos em que é legalmente admitida, deverá conter ou fazer-se acompanhar por este aviso. 


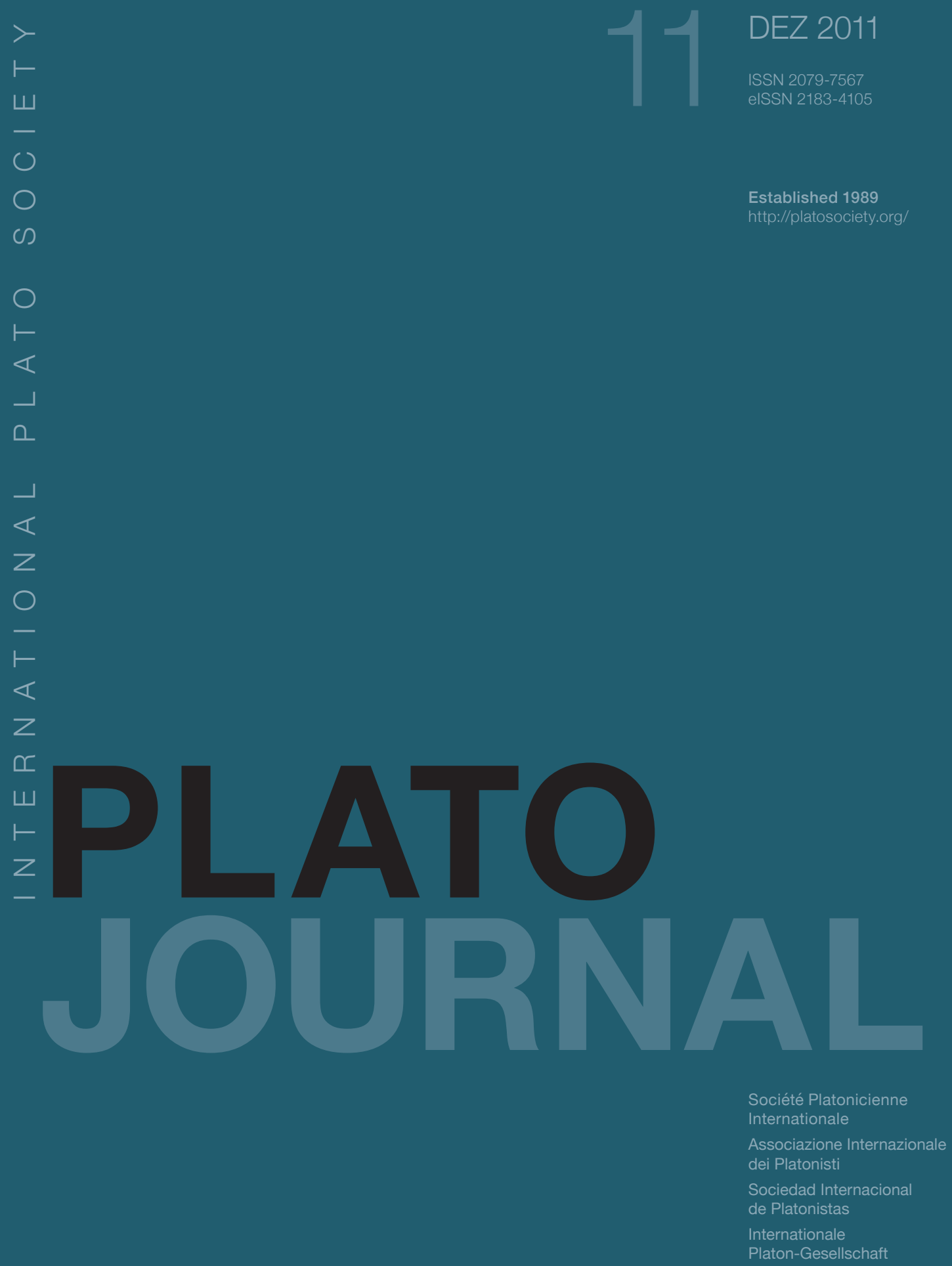




\title{
LE REMODELAGE POLITIQUE DE L'OIKOS DANS LA REPUBLIQUE \\ DE LA FAMILLE AU MODELE FAMILIAL, DE L'ECONOMIE DOMESTIQUE A L'ECONOMIE POLITIQUE
}

\author{
Étienne HELMER \\ Université de Porto Rico
}

\begin{abstract}
Beaucoup estiment que la cité juste élaborée par Platon dans la République se fonde sur l'abolition de l'oikos, terme désignant à la fois la famille et l'unité de production et de consommation des biens nécessaires à la vie. Pour Carlo Natali par exemple, Platon veut dans ce dialogue «éliminer la maison », et « dit explicitement que [...] l'abolition de l'oikos [...] découle de l'égalité des tâches des femmes $»^{1}$. Leo Strauss parle lui aussi de « l'abolition de la famille », tout comme Allan Bloom selon qui, au livre $\mathrm{V}$, « la famille est abolie $»^{2}$.
\end{abstract}

Toutefois, contrairement à ce qu'affirment ces commentateurs, l'abolition n'est pas le dernier mot de Platon sur l'oikos dans la République : il ne le fait pas disparaître de la cité juste mais le transforme. Pour comprendre cette transformation, il faut rappeler que l'oikos est à ses yeux le lieu d'un double attachement affectif, d'un double désir possessif tourné vers les membres de la famille d'un côté, vers la richesse accumulée dans et par l'oikos de l'autre. Ce sentiment est ambivalent: il met en jeu une force affective très puissante, qui pourrait servir de ciment à la cité si elle pouvait être transférée aux relations entre les citoyens; mais il favorise aussi la valorisation du particulier et du privé au détriment du commun, et constitue par là une source de conflits dans la société. Comment donc tirer parti de l'aspect positif de ce sentiment sans pâtir de son aspect négatif?

\footnotetext{
1 «L'élision de l'oikos dans la République de Platon », in Études sur la République, M. Dixsaut (dir.), avec la coll d'A. Larivée, Vol. I, Paris, Vrin, 2005, p. 221 et p. 213.

${ }^{2}$ La Cité et l'Homme, trad. fr., Paris, Le Livre de Poche, 2005, p. 264 ; La Cité et son Ombre. Essai sur la République de Platon, trad. fr., Paris, Le Félin, 2006, p. 127.
}

PLATO, The electronic Journal of the International Plato Society, n 11, 2011.

http://gramata.univ-paris1.fr/Plato/article98.html

(C) All rights of reproduction of any form reserved. 
Pour ce faire, Platon fait subir à l'oikos un remodelage qui consiste à séparer sa dimension familiale de sa fonction strictement économique, en transformant la première et en éliminant la seconde. D'une part, en effet, il n'abolit ni la structure ni la nature du lien familial propre à l'oikos, c'est-à-dire le lien de parenté et la relation affective forte qu'il comporte : il en déplace et en étend le champ d'application pour en faire un instrument d'unification politique. D'abord mobilisé à titre métaphorique pour penser les rapports entre les différents groupes de la cité au livre III, ce lien est ensuite utilisé au livre V comme un véritable modèle pour définir les rapports des gardiens entre eux et renforcer leur unité, dont dépend celle de la cité tout entière. Mais, d'autre part, pour que ce lien affectif puisse s'exercer pleinement à cette échelle, il faut qu'il soit exclusif de l'autre sentiment d'attachement propre à l'oikos, celui qui a pour objet la richesse matérielle et qui est générateur de concurrence et de discorde entre les oikoi particuliers. Le transfert du modèle familial au niveau politique suppose donc l'élimination de l'oikos comme unité de production économique privée, tournée vers l'expansion et la concurrence, au profit d'une économie pensée à l'aune de la cité tout entière.

Pour montrer comment, dans la République ${ }^{3}$, Platon convertit ainsi la force antipolitique de l'oikos en instrument d'unification de la cité juste, je rappellerai d'abord ce qu'était l'oikos à son époque, en soulignant ses deux dimensions, familiale et économique. J'exposerai ensuite les critiques qu'il lui adresse et en quoi il y voit une force antipolitique. Pour finir, j'expliquerai comment il le remodèle afin de le mettre au service de l'unité de la cité.

\section{L'oikos à l'époque de Platon}

À l'époque de Platon, l'oikos désigne à la fois le domaine agricole destiné à la satisfaction des besoins de la famille qui vit sur ce domaine, et cette famille ellemême, au sens large de «tous les gens de la maison avec leurs biens [...] », y compris les esclaves ${ }^{4}$. Ces deux composantes sont unies par la dimension morale de l'oikos comme foyer de valeurs régissant à la fois la production du nécessaire et les rapports humains. Pour comprendre le déplacement que Platon lui fait subir, il

\footnotetext{
${ }^{3}$ Malgré une solution différente, le projet est le même dans les Lois V, 739c1-d3.

${ }^{4}$ M. I. Finley, Le Monde d'Ulysse, [1956], Paris, Le Seuil, 2002, p. 69. Voir R.J. Littman, « Kinship in Athens $\gg$, Ancient Society, N¹0, 1979, p. 13.
}

PLATO, The electronic Journal of the International Plato Society, n 11, 2011.

http://gramata.univ-paris1.fr/Plato/article98.html

(C) All rights of reproduction of any form reserved. 
faut souligner trois de ses aspects, qui nous sont connus par deux sources principales : les recherches menées par des historiens contemporains sur la base de découvertes archéologiques, et l'Économique de Xénophon. Dans cet ouvrage, Socrate s'entretient d'abord directement avec Critobule (I-VI), puis lui rapporte un entretien qu'il a eu avec Ischomaque sur la meilleure façon d'administrer son oikos (VI-XXI). Sans condamner l'art oikonomique en tant que tel, Socrate pointe dans les propos d'Ischomaque les dangers potentiels que cet art recèle pour la vie éthique. Il faut donc supposer que la présentation qu'Ischomaque fait de l'oikos est couramment admise à l'époque pour que la pratique qui l'entoure fasse l'objet des remarques critiques de Socrate.

\section{S'enrichir}

Pour certains historiens, la finalité économique de l'oikos était bien souvent la richesse et non la simple subsistances. Le type de l'homo politicus forgé par M. Weber et utilisé par M.I. Finley pour penser «l'Homme grec » a contribué à occulter l'existence parallèle, dès l'époque classique, d'une rationalité économique tournée vers la recherche du profit, sans qu'il faille y voir pour autant une mentalité capitaliste ou une conduite oublieuse des valeurs éthiques : certains hommes agissaient en investisseurs rationnels, calculant, avant de se lancer, le rapport entre les risques liés à leurs projets et leurs bénéfices supposés. Si l'agriculture offrait de plus faibles profits que la vente et la location d'esclaves, et que l'exploitation des concessions minières, ces profits n'étaient pas négligeables pour autant, et l'oikos en constituait la base productive ${ }^{6}$.

Ce souci d'enrichissement apparaît aussi dans l'Économique. Comme Critobule, Ischomaque aime la richesse ${ }^{7}$. Il y voit un instrument lui permettant, « en rendant un culte magnifique aux dieux, en secourant [ses] amis dans le besoin, en contribuant de tout [son] pouvoir à l'embellissement de la ville $\gg^{8}$, de se gagner honnêtement une réputation de kalos kagathos. Ses efforts acharnés

5 «Economic Rationalism in Fourth-Century BCE Athens », P. Christesen, Greece \& Rome, Vol. 50, No. 1, April 2003, p. 31-56.

${ }^{6}$ Ibid. p. 46 et 52 .

${ }^{7} O E c$. XI, 8. Voir aussi dans ce sens les analyses de Leo Strauss, Xenophon's Socratic Discourse. An Interpretation of the Oeconomicus, Cornell University Press, Ithaca and London, 1970, p. 113, et 179-180.

${ }^{8} O E c$. XI. 9.

PLATO, The electronic Journal of the International Plato Society, n 11, 2011.

http://gramata.univ-paris1.fr/Plato/article98.html

(c) All rights of reproduction of any form reserved. 
pour accroître son oikos témoignent toutefois moins d'une quête de la vertu véritable, fondée sur l'intelligence, que d'une passion presque tyrannique pour la respectabilité sociale9. Aux yeux du Socrate de Xénophon comme de celui de Platon, une telle attitude révèle un manque de maîtrise de soi, et c'est donc une source bien fragile de la conduite éthique.

\section{Une polis miniature}

Selon Ischomaque, la différence entre la cité et l'oikos n'est pas de nature mais de degré : l'oikos est une cité miniature. Ainsi, un bon maître d'oikos doit s'inspirer des lois de Dracon et de Solon pour savoir comment diriger ses esclaves $^{10}$. La femme, elle, doit s'y conduire en « reine des abeilles » qui donne des ordres aux esclaves domestiques, et en « gardienne de ses lois » qui veille à la stricte application de ses prescriptions ${ }^{11}$.

Cette identité des communautés politique et domestique implique celle des pouvoirs qui s'y exercent. Selon Ischomaque, il y a en effet « une aptitude à commander commune à tous les genres d'activités, agriculture, politique, économie domestique, conduite de la guerre $\gg^{12}$. Le commandement est un art un, s'exerçant de la même façon dans tous les domaines, et dont le commandement militaire est le modèle ${ }^{13}$.

\section{La division sexuelle des tâches}

Enfin, à l'époque de Platon, l'oikos est organisé selon un principe de division sexuelle des tâches, fondé sur des motifs à la fois «naturels » et coutumiers: aux femmes les tâches intérieures, notamment le tissage, aux

9 G. Danzig, « Why Socrates was not a farmer. Xenophon's Oeconomicus as a Philosophical Dialogue », Greece \& Rome, vol. 50, N¹, 57-76, April 2003, en particulier p. 69.

${ }^{10} O E c$. XIV, 6-8.

${ }^{11} O E c$. VII, 16-43 ; IX, 14-15. L'image de l'abeille a dès cette époque une signification politique : par exemple Platon, Pol. $293 \mathrm{~d} 6$.

${ }^{12}$ CEc. XXI, 2. Voir aussi XIII, 5. Voir M. Foucault, Histoire de la sexualité, t. II, L'Usage des plaisirs, Paris, Gallimard, 1984, p. 200.

${ }^{13}$ Voir l'exemple de Cyrus et des officiers agricoles qui dépendent de lui, $O E c$. IV, 4-17 ; Cyr. VIII 1, 13-15; et la référence au commandement militaire pour les fonctions de femme dans l'oikos, IX, 15.

PLATO, The electronic Journal of the International Plato Society, n 11, 2011.

http://gramata.univ-paris1.fr/Plato/article98.html

(c) All rights of reproduction of any form reserved. 
hommes - outre la politique - les tâches extérieures, comme les travaux des champs, cette division ayant pour critère la force physique requise dans les deux $\operatorname{cas}^{14}$. Certains personnages de Platon partagent cette vision des choses: pour Ménon, « la vertu d'un homme consiste à être capable d'agir dans les affaires de la cité $[. .$.$] et la vertu d'une femme à bien gérer sa maison, à veiller à son intérieur, à$ le maintenir en bon état et à obéir à son mari $\gg^{15}$.

\section{L'oikos dans la République : une institution antipolitique}

Dans la République, Platon souligne la menace que représentent ces trois caractéristiques de l'oikos pour l'unité de la cité.

\section{La richesse de l'oikos contre l'unité de la polis}

Le désir de richesse animant l'oikos comporte, pour Platon, le risque d'être illimité, de manquer de mesure. Cet appétit est en effet la manifestation la plus répandue de la pleonexia, cette tendance concurrentielle à vouloir avoir davantage que les autres et que « toute nature poursuit comme un bien $»^{16}$, comme l'illustre l'histoire de Gygès ${ }^{17}$. Il est donc difficilement compatible avec la modération que réclame la conduite éthique ${ }^{18}$, et aboutit nécessairement au conflit des intérêts privés, à la disparition de l'idée même d'intérêt commun, et à la dissension (stasis) dans la cité : «car l'intérêt général rassemble, tandis que l'intérêt particulier déchire les cités ${ }^{19}$. 》

\footnotetext{
${ }^{14} O E c$. VII, 22-23.

${ }^{15}$ Men. 71e1-7.

${ }^{16}$ Rép. II, 359c4-5. Voir J. Gutglueck, « From pleonexia to polupragmosunè. A conflation of possession and action in Plato's Republic 349b-350c », American Journal of Philology, vol. 109, $\mathrm{N}^{\circ} 1,1988$, p. 20-39, en particulier, p. 25-26.

${ }^{17}$ Rép. II, 359b-360d.

${ }^{18}$ Rép. IV, 430d sq.

${ }^{19}$ Lois, IX, 875a1-6.
}

PLATO, The electronic Journal of the International Plato Society, n 11, 2011.

http://gramata.univ-paris1.fr/Plato/article98.html

(c) All rights of reproduction of any form reserved. 
Ce danger est encore plus grand quand il s'agit des futurs dirigeants de la cité. Le souci de l'oikos corrompt leur engagement dans la politique véritable, celle qui repose sur la philosophie et se soucie de l'intérêt commun. Cette corruption des gardiens peut se produire durant leur période de formation :

« ceux qui s'attachent à présent à la philosophie, et qui sont des adolescents à peine sortis de l'enfance, attendant le moment de s'occuper de la gestion de la maison (oikonomia) et de gagner de l'argent, la quittent à peine ils se sont approchés de la partie la plus difficile de cette activitée ${ }^{20}$. »

Mais elle peut aussi survenir durant l'exercice de leurs fonctions, entrainnant ainsi leur propre désastre et celui de toute la cité :

« dès qu'eux-mêmes auront acquis un terrain privé (gèn idian), des maisons (oikias), et des monnaies en usage, ils seront administrateurs de maisons (oikonomoi) et cultivateurs, au lieu d'être des gardiens, et ils deviendront les maîtres hostiles et non plus les alliés des autres citoyens ${ }^{21}$. »

\section{Lapolis n'est pas un grand oikos}

La véritable polis ne peut donc, pour Platon, être vue comme un oikos à grande échelle, ni le bon politique assimilé à un oikonomos tel que l'a défini Ischomaque. La meilleure cité est en effet pour Platon celle dont l'unité est la plus grande, et dont les citoyens partagent des valeurs communes grâce au travail d'unification réalisé par l'art politique authentique. Socrate préconise ainsi que la cité s'accroisse «tant que, en s'accroissant, elle persiste à être une, mais pas audelà », de sorte qu'elle ne soit «ni petite ni apparemment grande, mais qu'elle soit en quelque sorte suffisante et une $\gg^{22}$. A ses yeux, on ne gouverne donc pas la cité comme on administre un domaine: l'oikos est une institution fondée sur l'accroissement et fomentant la division, alors que la polis juste réclame l'unité et la communauté.

${ }^{20}$ Rép. VI, 497e6-498b1.

${ }^{21}$ Rép. III, 417a6-b5.

${ }^{22}$ Rép. IV, 423b9-10 ; c3-4. Cf. le paradigme de l'unification du tissu politique dans le Politique, 310e5-311a2.

PLATO, The electronic Journal of the International Plato Society, n 11, 2011.

http://gramata.univ-paris1.fr/Plato/article98.html

(c) All rights of reproduction of any form reserved. 


\section{La famille et les femmes contre la cité}

Enfin, on l'a vu, l'oikos est aux yeux de Platon un lieu affectif où s'élabore un sentiment d'attachement exclusif au privé et au particulier, qui a pour objet aussi bien les choses que les membres de la famille, et qui se construit contre son contraire, contre le sentiment d'appartenance à une communauté dépassant les intérêts particuliers ${ }^{23}$. C'est pourquoi Socrate estime nécessaire de trouver une solution pour préserver la cité « de la dissension interne, de tout ce qui, à cause de la possession de biens matériels, d'enfants et de parents fait que des hommes sont en dissension $\gg{ }^{24}$. Ce repli sur soi dont l'oikos est le centre est très net, par exemple, chez les timocrates qui, pour jouir de leurs richesses en privé, se retranchent derrière les enceintes de leurs maisons ${ }^{25}$. Plus généralement, il est le fait de tous les acteurs domestiques, et des femmes en particulier qui, dans les cités mal constituées, n’ont pas accès à la vie publique et sont confinées dans les maisons en raison de la division des tâches exposée plus haut. Aussi ce mouvement centripète qu'elles initient se double-t-il d'un mouvement centrifuge : soustraites au monde commun de la polis, elles ne l'envisagent que comme une extension du monde privé et contribuent ainsi à sa ruine, comme lorsqu'elles incitent fils et maris à s'enrichir et à étendre l'oikos au détriment de la polis afin d'en retirer pour ellesmêmes une plus grande visibilité sociale ${ }^{26}$.

\section{L’oikos politisé de la République}

En tant qu'unité économique et que famille, l'oikos, on le voit, est l'ennemi de la cité juste. Mais Platon ne l'abolit pas pour autant de la République: il le transforme en un instrument d'unification politique, en détournant sa force d'accaparement matériel et affectif au profit de la cité. Il lui ôte pour cela sa fonction économique et ne lui conserve, en l'étendant, que sa dimension familiale.

${ }^{23}$ Dans les Lois, pour résoudre cette même difficulté, le législateur invite les citoyens à se considérer comme appartenant à la cité avant d'appartenir à leur famille, Lois VII, 804d5-6 ; XI, 923a6-b1.

${ }^{24}$ Rép. V, 464d9-e2. Je souligne.

${ }^{25}$ Rép. VIII, 548a5-b3.

${ }^{26}$ Rép. VIII, 549c8-e1.

PLATO, The electronic Journal of the International Plato Society, n 11, 2011.

http://gramata.univ-paris1.fr/Plato/article98.html

(c) All rights of reproduction of any form reserved. 


\section{La richesse : limites et interdiction}

En ce qui concerne le groupe des producteurs de la cité de la République, on peut supposer, faute d'indications précises à leur sujet, que leur enrichissement sera limité dans la cité juste, au nom de son unitée ${ }^{27}$. Le silence sur l'oikos dans le récit de la naissance économique de la cité laisse penser aussi que Platon transfère les fonctions économiques à la cité précisément pour empêcher cette expansion concurrentielle des $o i k o i^{28}$.

Les choses sont plus claires avec les gardiens, auxiliaires ou dirigeants. Leur unité conditionnant celle de la cité tout entière, il leur est interdit de posséder quoi que ce soit, pour empêcher dans leur groupe les débordements conflictuels du désir de richesse. Ils doivent se plier à une vie matérielle commune ${ }^{29}$, et recevoir leur « salaire », c'est-à-dire leur subsistance, de la part du groupe des producteurs ${ }^{30}$, ce qui revient à les soustraire à l'oikos comme unité économique de production et de consommation.

\section{La famille : métaphore puis modèle des relations politiques}

Les choses sont plus complexes avec l'oikos envisagé comme famille. La référence à la famille apparaît une première fois dans l'épisode du « noble mensonge » : tous les membres de la cité sont frères car ils sont nés d'une même mère, la terre ${ }^{31}$. Pour trois raisons, cette référence est plutôt une image ou une métaphore qu'un modèle stricto sensu projeté sur la citééci ${ }^{32}$ Cette référence à la famille est d'abord incomplète, la place du père n'étant pas évoquée, et l'on voit mal qui pourrait la remplir. Elle est ensuite provisoire, la cité entière étant, plus tard dans le texte, comparée à un individu ${ }^{33}$. Elle est enfin symbolique, la « terre mère » renvoyant au thème classique de l'autochtonie. Il ne s'agit donc pas de dire ici que la société est une grande famille, comme ce sera le cas chez les gardiens, mais d'inviter ses membres à faire comme s'ils en étaient une. Cette référence à la

\footnotetext{
${ }^{27}$ Voir la citation correspondant à la note 22.

${ }^{28}$ Rép. II, 369b-373d. Dans les Lois, la production a lieu dans chacun des 5040 lots, mais ils ne produisent pas pour eux-mêmes mais pour toute la cité, VIII, 847e2-848c6.

${ }^{29}$ Rép. III, 416d3-417a6.

${ }^{30}$ Rép. IV, 463a10-b3.
}

PLATO, The electronic Journal of the International Plato Society, n 11, 2011.

http://gramata.univ-paris1.fr/Plato/article98.html

(c) All rights of reproduction of any form reserved. 
famille a pour but de préserver l'unité de la cité malgré la nécessaire hiérarchie des groupes et la distribution des individus dans chacun de ces groupes. La parenté (suggeneia) politique de tous les citoyens ${ }^{34}$ n'est donc pas à prendre ici au sens propre, mais en référence au lien affectif fort qu'elle évoque : en dépit de leurs différences de fonction, les citoyens doivent nourrir entre eux des sentiments fraternels pour rester unis.

Avec le groupe des gardiens en revanche, Socrate utilise la force du lien affectif de l'oikos et sa structure de parenté comme véritable modèle de leurs relations, avec cette différence majeure - qui constitue le déplacement que Platon lui fait subir - que les liens de parenté traditionnels ne s'appliquent plus à des individus mais à des générations entières, au point que les mères ne pourront reconnaître leurs enfants biologiques ${ }^{35}$ :

« À compter du jour où l'un d'eux deviendra un promis, les rejetons qui naîtront entre le huitième et le dixième mois après, tous ceux-là il les appellera, les mâles ses fils, et les femelles ses filles, et eux le nommeront père, et de la même façon leurs rejetons il les nommera petits-enfants et eux à leur tour le nommeront grand-père (et grand-mère); et ceux qui seront nés pendant le moment où leurs mères et pères engendraient se nommeront sœurs et frères : et par conséquent, comme nous le disions à l'instant, ils ne toucheront pas les uns aux autres ${ }^{36}$. »

Les gardiens forment donc une grande famille, structurée autour de la prohibition de l'inceste. Mais c'est une famille désormais étendue, au sens où les relations affectives liées à la parenté y sont désindividualisées et élargies avec la parenté elle-même : le sentiment de possession affective qui attache un individu à un autre y est dorénavant impossible, sa force étant détournée au profit d'un sentiment de familiarité à l'échelle du groupe entier des gardiens. Dans la cité juste, la communauté matérielle des gardiens se double ainsi d'une communauté

${ }^{31}$ Rép. III, 414d1-415c7.

${ }^{32}$ V. Goldschmidt, Le Paradigme dans la dialectique platonicienne, [1947], Paris, Vrin, 1985, p. 111-117.

${ }^{33}$ Rép. V, 462c10 sq.

${ }^{34}$ Rép. III, $415 \mathrm{a} 7$.

${ }^{35}$ Rép. V, 460c8-d1.

${ }^{36}$ Rép. V, 461d2-e2.

PLATO, The electronic Journal of the International Plato Society, n 11, 2011.

http://gramata.univ-paris1.fr/Plato/article98.html

(c) All rights of reproduction of any form reserved. 
familiale : il faut que « les femmes y soient toutes communes à tous les hommes, et qu'aucune ne vive en privé avec aucun; que les enfants eux aussi soient communs, et qu'un parent ne connaisse pas son propre rejeton, ni un enfant son parent $\gg^{37}$.

Les gardiens forment ainsi une famille unique, au double sens du terme : c'est une famille une ou unie, et c'est la seule famille, et c'est précisément parce qu'elle est la seule qu'elle est une, la rivalité entre oikoi dénoncée par Platon dans les cités de son temps étant désormais impossible dans cette configuration nouvelle. Cette famille est ainsi rendue politique au sens où sa structure, loin de nuire à la cité, est enfin compatible avec elle et même la favorise, l'unité des gouvernants conditionnant celle de toute la cité $e^{38}$.

\section{Politiser les femmes}

La vie commune des gardiens organisée sur le modèle d'une famille suit la «vague » qui fait accéder les femmes à la politique, en faisant d'elles des gardiennes au même titre que les hommes ${ }^{39}$. En éliminant ainsi la division des tâches économiques exposée par Ischomaque, et les dangers politiques que comportait la réclusion domestique des femmes, Platon élimine donc complètement l'économie de l'oikos des gardiens.

\section{Conclusion}

Modeler toute la cité sur l'oikos serait pour Platon entraîner la cité à sa perte. Ce que Platon veut abolir dans la République, ce n'est toutefois pas tant l'oikos que le sentiment antipolitique de clôture et d'accaparement dont il est la source. Il y parvient en étendant le sentiment d'affection familiale entre individus à une plus grande échelle dans le groupe des gardiens, et en transférant l'économie de la sphère domestique à la cité tout entière. En d'autres termes, Platon maintient le modèle familial de l'oikos dans la cité en rendant, à mots couverts, l'économie politique.

\footnotetext{
${ }^{37}$ Rép. V, 457c10-d3.

${ }^{38}$ Leur discorde provoquera la dégénérescence du bon régime politique, Rép. VIII, $547 \mathrm{a}$-c.

${ }^{39}$ Rép. V, 451c-457b. Voir aussi Lois VII, 804d6-806d3 ; 813e3-814c5.
}

PLATO, The electronic Journal of the International Plato Society, n 11, 2011.

http://gramata.univ-paris1.fr/Plato/article98.html

(c) All rights of reproduction of any form reserved. 


\section{BIBLIOGRAPHIE}

Bloom A. : La Cité et son Ombre. Essai sur la République de Platon, trad. fr., Paris, Le Félin, 2006

Christesen P.: « Economic Rationalism in Fourth-Century BCE Athens », Greece \& Rome, Vol. 50, No. 1, April 2003, p. 31-56

DANZIG G. : « Why Socrates was not a farmer. Xenophon's Oeconomicus as a Philosophical Dialogue », Greece \& Rome, vol. 50, №1, p. 57-76

FinLEY M.I. : Le Monde d'Ulysse, [1956], Paris, Le Seuil, 2002

FouCAult M. : Histoire de la sexualité, t. II, L'Usage des plaisirs, Paris, Gallimard, 1984

GOLDSCHMIDT V. : Le Paradigme dans la dialectique platonicienne, [1947], Paris, Vrin, 1985

GUTGLUECK J. : « From pleonexia to polupragmosunè. A conflation of possession and action in Plato's Republic 349b-350c », American Journal of Philology, vol. 109, $\mathrm{N}^{\circ} 1,1988$, p. 20-39

LiTTMAn R.J. : « Kinship in Athens », Ancient Society, N¹0, 1979, p. 5-31

NATALi C. : «L'élision de l'oikos dans la République de Platon », in Études sur la République, M. Dixsaut (dir.), avec la coll d'A. Larivée, Vol. I, Paris, Vrin, 2005, p. 199-223

Pomeroy S.B.: Families in classical and Hellenistic Greece. Representations and realities, Oxford, Clarendon press, 1997

Schofield M. : Plato, Oxford, New York, Oxford University Press, 2006

STRAuSS L.: Xenophon's Socratic Discourse. An Interpretation of the Oeconomicus, Cornell University Press, Ithaca and London, 1970

Strauss L. : La Cité et l'Homme, trad. fr., Paris, Le Livre de Poche, 2005

PLATO, The electronic Journal of the International Plato Society, n 11, 2011.

http://gramata.univ-paris1.fr/Plato/article98.html

(c) All rights of reproduction of any form reserved. 